\title{
ON BALANCED PRODUCTS OF DISTRIBUTIONS IN COLOMBEAU ALGEBRA
}

\author{
Blagovest P. Damyanov \\ Institute for Nuclear Researches and Nuclear Energy \\ Bulgarian Academy of Sciences \\ 72, Bul. Tzarigradsko Shosse, Sofia, 1784, BULGARIA
}

\begin{abstract}
Results on singular products of Schwartz distributions on the Euclidean space $\mathbb{R}^{m}$ are derived when the products are so 'balanced' that they exist in the distribution space. The results follow the idea of a known distributional product introduced by Jan Mikusiński and are obtained in the Colombeau algebra of generalized functions. This algebra contains the distributions and the notion of 'association' permits obtaining results on the level of distributions.
\end{abstract}

AMS Subject Classification: 46F30, 46F10

Key Words: Schwartz distributions, multiplication, Colombeau generalized functions

The Schwartz distributions are widely employed in natural sciences and many other mathematical fields. Since products of distributions with coinciding singularities often appear in them, the problem of their multiplication has been objective of studies for a long time. On the other hand, the differential algebra $\mathcal{G}$ of generalized functions of J.F. Colombeau [1] has become popular since it has almost optimal properties for tackling non-linear problems of Schwartz distributions: they are linearly embedded in $\mathcal{G}$ and the multiplication is compatible with differentiation and products with smooth functions. The so-called 'association' in $\mathcal{G}$, being a faithful generalization of the equality of distributions, 
makes it possible to obtain results on the level of distributions. This is why the generalized functions of Colombeau have application to various mathematical fields; see for example the recent paper [6]. Here we follow the above approach: products of distributions with coinciding point singularities are evaluated, as embedded in $\mathcal{G}$, and their product admits an associated distribution.

Recall now the famous result of Jan Mikusiński published in [5]:

$$
x^{-1} \cdot x^{-1}-\pi^{2} \delta(x) \cdot \delta(x)=x^{-2}, \quad x \in \mathbb{R} .
$$

Although, none of the products on the left-hand side here exists, their difference is so 'balanced' that it has a correct meaning. Balanced formulas of this kind can be found in the mathematical and physical literature. In [2] we derived generalizations of $(1)$ in the Colombeau algebra $\mathcal{G}(\mathbb{R})$, while in [3] and [4] various balanced formulas were obtained for singular products of distributions.

In this paper, new results on singular products of the distributions $x_{ \pm}^{a}$ and $\delta^{(p)}(x) \quad\left(x \in \mathbb{R}^{m}\right)$ are given in the algebra $\mathcal{G}\left(\mathbb{R}^{m}\right)$. We first remind the basics of the Colombeau theory.

Notation 1(a) If $\mathbb{N}_{0}$ stands for the nonnegative integers and $p=\left(p_{1}, p_{2}, \ldots\right.$, $\left.p_{m}\right)$ is a multiindex in $\mathbb{N}_{0}^{m}$, we let $|p|=\sum_{i=1}^{m} p_{i}$ and $p !=p_{1} ! \ldots p_{m} !$. If $x=$ $\left(x_{1}, \ldots, x_{m}\right) \in \mathbb{R}^{m}$, we denote $x^{p}=\left(x_{1}^{p_{1}}, x_{2}^{p_{2}}, \ldots, x_{m}^{p_{m}}\right)$ and $\partial^{p}=\partial^{|p|} / \partial x_{1}^{p_{1}} \ldots \partial x_{m}^{p_{m}}$.

(b) If $q \in \mathbb{N}_{0}$, we put $A_{q}(\mathbb{R})=\left\{\varphi(x) \in \mathcal{D}(\mathbb{R}): \int_{\mathbb{R}} x^{j} \varphi(x) d x=\delta_{0 j}\right.$ for $0 \leq j \leq q$, where $\delta_{00}=1, \delta_{0 j}=0$ for $\left.j>0\right\}$. This also extends to $\mathbb{R}^{m}$ as an $m$-fold product: $A_{q}\left(\mathbb{R}^{m}\right)=\left\{\varphi(x) \in \mathcal{D}\left(\mathbb{R}^{m}\right): \varphi\left(x_{1}, \ldots, x_{m}\right)=\prod_{i=1}^{m} \chi\left(x_{i}\right)\right.$ for some $\chi$ in $\left.A_{q}(\mathbb{R})\right\}$. Finally, we denote $\varphi_{\varepsilon}=\varepsilon^{-m} \varphi\left(\varepsilon^{-1} x\right)$ for $\varphi$ in $A_{q}\left(\mathbb{R}^{m}\right)$ and $\varepsilon>0$.

Definition 1. Let $\mathcal{E}\left[\mathbb{R}^{m}\right]$ be the algebra of functions $f(\varphi, x): A_{q}\left(\mathbb{R}^{m}\right) \times$ $\mathbb{R}^{m} \rightarrow \mathbb{C}$ that are infinitely differentiable, by a fixed 'parameter' $\varphi$. The generalized functions of Colombeau are elements of the quotient algebra $\mathcal{G} \equiv$ $\mathcal{G}\left(\mathbb{R}^{m}\right)=\mathcal{E}_{\mathrm{M}}\left[\mathbb{R}^{m}\right] / \mathcal{J}\left[\mathbb{R}^{m}\right]$. Here $\mathcal{E}_{\mathrm{M}}\left[\mathbb{R}^{m}\right]$ is the subalgebra of functions that for each compact subset $K$ of $\mathbb{R}^{m}$ and $p \in \mathbb{N}_{0}^{m}$ there is a $q \in \mathbb{N}$ so that for any $\varphi \in A_{q}\left(\mathbb{R}^{m}\right), \sup _{x \in K}\left|\partial^{p} f\left(\varphi_{\varepsilon}, x\right)\right|=O\left(\varepsilon^{-q}\right)$, as $\varepsilon \rightarrow 0_{+}$. The ideal $\mathcal{J}\left[\mathbb{R}^{m}\right]$ of $\mathcal{E}_{\mathrm{M}}\left[\mathbb{R}^{m}\right]$ is the set of functions that for each compact subset $K$ of $\mathbb{R}^{m}$ and $p \in \mathbb{N}_{0}^{m}$ there is a $q \in \mathbb{N}$ so that for every $r \geq q$ and $\varphi \in A_{r}\left(\mathbb{R}^{m}\right)$, $\sup _{x \in K}\left|\partial^{p} f\left(\varphi_{\varepsilon}, x\right)\right|=O\left(\varepsilon^{r-q}\right)$, as $\varepsilon \rightarrow 0_{+}$.

The algebra $\mathcal{G}$ contains the distributions, canonically embedded as a $\mathbb{C}$ vector subspace, by the map $i: \mathcal{D}^{\prime}\left(\mathbb{R}^{m}\right) \rightarrow \mathcal{G}: u \mapsto \widetilde{u}=\{\widetilde{u}(\varphi, x)=(u * \check{\varphi})(x)\}$, where $\check{\varphi}(x)=\varphi(-x)$ and $\varphi \in A_{q}\left(\mathbb{R}^{m}\right)$.

Definition 2(a). The functions $f, g \in \mathcal{G}$ are 'associated', which is denoted as $f \approx g$, if for some $f\left(\varphi_{\varepsilon}, x\right)$ and $g\left(\varphi_{\varepsilon}, x\right)$ and arbitrary $\psi(x) \in \mathcal{D}\left(\mathbb{R}^{m}\right)$ there is some $q \in \mathbb{N}_{0}$ so that, for any $\varphi(x) \in A_{q}(\mathbb{R})$, it holds $\lim _{\varepsilon \rightarrow 0_{+}} \int_{\mathbb{R}}\left[f\left(\varphi_{\varepsilon}, x d x\right)\right.$ - 
$\left.g\left(\varphi_{\varepsilon}, x\right)\right] \psi(x) d x=0$.

(b) A function $f \in \mathcal{G}$ has some $u \in \mathcal{D}^{\prime}\left(\mathbb{R}^{m}\right)$ as 'associated distribution', denoted $f \approx u$, if for some representative $f\left(\varphi_{\varepsilon}, x\right)$ of $f$ and arbitrary $\psi(x) \in \mathcal{D}\left(\mathbb{R}^{m}\right)$ there is a $q \in \mathbb{N}_{0}$ such that, for any $\varphi(x) \in A_{q}(\mathbb{R})$, it holds $\lim _{\varepsilon \rightarrow 0_{+}} \int_{\mathbb{R}} f\left(\varphi_{\varepsilon}, x\right) \psi(x) d x=\langle u, \psi\rangle$. The distribution associated, if it exists, is unique and its image is associated with the former [1], the association thus being a faithful generalization of equality in $\mathcal{D}^{\prime}\left(\mathbb{R}^{m}\right)$. Then, by product of distributions in the algebra $\mathcal{G}$ is meant the product of their embeddings, whenever the result admits associated distribution. This interpretation can be given: reducing the information to distributional level, the product behaves like the distribution associated.

Now, if $\widetilde{x_{+}^{p}}$ and $\widetilde{\delta^{(p+1)}}(x)$ are embeddings in $\mathcal{G}(\mathbb{R})$ of the corresponding distributions, this generalization of (1) for any $p, q \in \mathbb{N}$ was proved in [2]:

$$
\widetilde{x^{-p}} \cdot \widetilde{x^{-q}}-\frac{(-1)^{p+q} \pi^{2}}{(p-1) !(q-1) !} \widetilde{\delta(p-1)}(x) \cdot \widetilde{\delta^{(q-1)}}(x) \approx x^{-p-q}, x \in \mathbb{R} .
$$

Below we shall need this general property of balanced products of distributions on $\mathbb{R}^{m}$ with tensor-product structure.

Theorem 1. Let $u_{k}, v_{k}, k=1,2$ be distributions in $\mathcal{D}^{\prime}\left(\mathbb{R}^{m}\right)$ such that $u_{k}(x)=\prod_{i=1}^{m} u_{k}^{i}\left(x_{i}\right), v_{k}(x)=\prod_{i=1}^{m} v_{k}^{i}\left(x_{i}\right)$, where all $u_{k}^{i}, v_{k}^{i}$ are distributions in $\mathcal{D}^{\prime}(\mathbb{R})$, and their embeddings in $\mathcal{G}(\mathbb{R})$ satisfy: $\widetilde{u_{1}^{i}} \cdot \widetilde{v_{1}^{i}}-\widetilde{u_{2}^{i}} \cdot \widetilde{v_{2}^{i}} \approx 0, \quad i=$ $1, \ldots, m$. Then it holds for the embeddings in $\mathcal{G}\left(\mathbb{R}^{m}\right)$ of the tensor-product distributions $u_{k}, v_{k}$ :

$$
\widetilde{u_{1}} \cdot \widetilde{v_{1}}-\widetilde{u_{2}} \cdot \widetilde{v_{2}} \approx 0 \text {. }
$$

Proof. It holds in $\mathcal{G}(\mathbb{R}): \widetilde{u_{1}^{i}} \cdot \widetilde{v_{1}^{i}} \approx \widetilde{u_{2}^{i}} \cdot \widetilde{v_{2}^{i}}$ for $i=1, \ldots m$. We restrict ourselves to the subspace of functions $\psi(x)=\prod_{i=1}^{m} \psi_{i}\left(x_{i}\right)$, with each $\psi_{i} \in \mathcal{D}(\mathbb{R})$. Due to the tensor-product structure of $u_{k}, v_{k} \in \mathcal{D}^{\prime}\left(\mathbb{R}^{m}\right)$ and $\varphi \in A_{q}\left(\mathbb{R}^{m}\right)$, on applying a Fubini-type theorem for tensor-product distributions, we get:

$$
F=\left\langle\widetilde{u_{2}}\left(\varphi_{\varepsilon}, x\right) \widetilde{v_{2}}\left(\varphi_{\varepsilon}, x\right), \psi(x)\right\rangle+o(1) .
$$

Here, the Landau symbol $o$ (1) stands for any function that tends to 0 , as $\varepsilon \rightarrow 0$. Therefore,

$$
\lim _{\varepsilon \rightarrow 0}\left\langle\left[\widetilde{u_{1}}\left(\varphi_{\varepsilon}, x\right) \widetilde{v_{1}}\left(\varphi_{\varepsilon}, x\right)-\widetilde{u_{2}}\left(\varphi_{\varepsilon}, x\right) \widetilde{v_{2}}\left(\varphi_{\varepsilon}, x\right)\right], \psi(x)\right\rangle=0 .
$$

The set of functions $\psi(x)=\prod_{i=1}^{m} \psi_{i}\left(x_{i}\right)$ is dense subset of $\mathcal{D}\left(\mathbb{R}^{m}\right)$, thus by Definition 2 (b), the tensor-product distributions satisfy (2). This completes the proof. 
We proceed to evaluating some balanced products of the distributions $x_{ \pm}^{a}$ and $\delta^{(p)}(x)$ in $\mathcal{G}\left(\mathbb{R}^{m}\right)$.

Notation 2 (a) Let $a=\left(a_{1}, \ldots, a_{m}\right)$ be ordered $m$-tuples in $\mathbb{R}^{m}$ with vector operations and $k \in \mathbb{Z}$. Then $a+k$ stands for $\left(a_{1}+k, \ldots, a_{m}+k\right), a>k$ denotes $a_{i}>k$ for $i=1, \ldots, m$. We use the short-hand notations $x^{a}=\left(x_{1}^{a_{1}}, \ldots, x_{m}^{a_{m}}\right)$ and $\Gamma(a)=\prod_{i=1}^{m} \Gamma\left(a_{i}\right)$. Finally, $\Omega=\{a \in \mathbb{R}: a \neq-1,-2, \ldots\}$ and $\Omega^{m}$ is the $m$-fold tensor product of $\Omega$.

(b) Denote further the 'normed' powers of $x \in \mathbb{R}^{m}, a \in \Omega^{m}$ and supported only in one quadrant of $\mathbb{R}^{m}$, as follows : vskip -10pt

$$
\chi_{ \pm}^{a} \equiv \chi_{ \pm}^{a}(x)=\left\{\frac{ \pm x^{a}}{\Gamma(a+1)} \text { for } x>0 / x<0, \quad=0 \text { elsewhere }\right\} .
$$

By Notation $1(\mathrm{a}), x^{a} / \Gamma(a+1)=\prod_{i=1}^{m} x_{i}^{a_{i}} / \Gamma\left(a_{i}\right)$. Now we shall prove this.

Theorem 2. If $a, b \in \Omega^{m}$ and $b>-2$, then for each $p \in \mathbb{N}_{0}^{m}$ the following balanced products hold in $\mathcal{G}\left(\mathbb{R}^{m}\right)$ :

$$
\begin{aligned}
& \widetilde{\chi_{+}^{a}} \cdot \widetilde{\chi_{-}^{b-a}}-\widetilde{\chi_{-}^{b+p+1}} \cdot \widetilde{\delta(p)}(x) \approx 0, \\
& \widetilde{\chi_{+}^{a}} \cdot \widetilde{\chi_{-}^{b-a}}-(-1)^{|p|} \widetilde{\chi_{+}^{b+p+1}} \cdot \widetilde{\delta(p)}(x) \approx 0 .
\end{aligned}
$$

Proof. In dimension 1 , since $x \mapsto \chi_{ \pm}^{a}(x)$ is locally-integrable function for $a>-1$, we define the distribution $\chi_{ \pm}^{a}$ for each $a \in \Omega$, if for some $k \in \mathbb{N}_{0}$ such that $a+k+1>0$, we put: $\chi_{ \pm}^{a}=\partial_{x}^{k} \chi_{+}^{a+k}=( \pm 1)^{k} \partial_{x}^{k} x_{ \pm}^{a+k} / \Gamma(a+k+1)$. If $k \in \mathbb{N}_{0}$ satisfies the condition $k+1>\max \{-a,-b,-a-b-1\}$, we get for the embedding in $\mathcal{G}$ :

$$
\widetilde{\chi_{+}^{a}}\left(\varphi_{\varepsilon}, x\right)=\frac{(-1)^{k} \varepsilon^{-k}}{\Gamma(a+k+1)} \int_{-x / \varepsilon}^{l}(x+\varepsilon u)^{a+k} \varphi^{(k)}(u) d u .
$$

We assumed here that $\sup \varphi(x) \subseteq[-l, l]$ for some $l \in \mathbb{R}_{+}$and made the substitution $u=(y-x) / \varepsilon$. The same applies for $\widetilde{\chi_{-}^{b-a}}\left(\varphi_{\varepsilon}, x\right)$. If $\psi(x) \in \mathcal{D}(\mathbb{R})$, denote by $F_{1}(\varepsilon):=\left\langle\widetilde{\chi_{+}^{a}}\left(\varphi_{\varepsilon}, x\right) \widetilde{\chi_{-}^{b-a}}\left(\varphi_{\varepsilon}, x\right), \psi(x)\right\rangle$ and $G(\varepsilon)=\Gamma(a+k+1) \Gamma(b+k+$ 1) $F_{1}(\varepsilon)$.

Further, by the Taylor theorem, $\psi(-\varepsilon w)=\psi(0)-(\varepsilon w) \psi^{\prime}(\eta w), \eta \in(0,1)$. Then on changing twice the order of integration and applying the substitution $w \rightarrow t=(w-v) /(u-v)$ we obtain

$$
G(\varepsilon)=(-1)^{k} \psi(0) \varepsilon^{b+1} \int_{-l}^{l} \varphi^{(k)}(u) \int_{-l}^{u}(u-v)^{b+2 k+1} \varphi^{(k)}(v) d v d u
$$




$$
\times \int_{0}^{1}(1-t)^{b+k} t^{b+k} d t+o(1)
$$

Then, applying the definition of Euler integral and the rule for differentiating integrals we get:

$$
F_{1}(\varepsilon)=\frac{\psi(0) \varepsilon^{b+1}}{\Gamma(b+k+2)} \int_{-l}^{l} \varphi(u) \int_{-l}^{u}(u-v)^{b+k+1} \varphi^{(k)}(v) d v d u+o(1) .
$$

In the one-variable case, for arbitrary $\psi(x) \in \mathcal{D}(\mathbb{R})$ and $n \in \mathbb{N}_{0}$, consider next $F_{2}(\varepsilon)=\left\langle\widetilde{\chi_{-}^{b+p+1}}\left(\varphi_{\varepsilon}, x\right) \widetilde{\delta(p)}\left(\varphi_{\varepsilon}, x\right), \psi(x)\right\rangle$. On changing the variables and applying the Taylor theorem, we have

$$
F_{2}(\varepsilon)=\frac{(-1)^{p} \psi(0) \varepsilon^{b+1}}{\Gamma(b+p+2)} \int_{-l}^{l} \varphi^{(p)}(u) \int_{-l}^{u}(u-v)^{b+p+1} \varphi(v) d v d u+o(1) .
$$

In view of $\left.T(u, v)\right|_{v=u}=0$ and $a+b>-2$, we get

$$
F_{2}(\varepsilon)=\frac{\psi(0) \varepsilon^{b+1}}{\Gamma(b+2)} \int_{-l}^{l} \varphi(u) \int_{-l}^{u}(u-v)^{b+1} \varphi(v) d v d u+o(1) .
$$

Thus we obtain for $F_{2}(\varepsilon):=\left\langle\widetilde{\chi_{+}^{a}}\left(\varphi_{\varepsilon}, x\right) \widetilde{\chi_{-}^{b-a}}\left(\varphi_{\varepsilon}, x\right), \psi(x)\right\rangle$ :

$$
F_{2}(\varepsilon)=\frac{\psi(0) \varepsilon^{b+1}}{\Gamma(b+2)} \int_{-l}^{l} \varphi(u) \int_{-l}^{u}(u-v)^{b+1} \varphi(v) d v d u+o(1) .
$$

The results for $F_{1}(\varepsilon)$ and $F_{2}(\varepsilon)$ give $F_{1}(\varepsilon)-F_{2}(\varepsilon)=o(1)$, and thus

$$
\lim _{\varepsilon \rightarrow 0}\left\langle\widetilde{\chi_{+}^{a}}\left(\varphi_{\varepsilon}, x\right) \widetilde{\chi_{-}^{b-a}}\left(\varphi_{\varepsilon}, x\right)-\widetilde{\chi_{-}^{b+p+1}}\left(\varphi_{\varepsilon}, x\right) \widetilde{\delta(p)}\left(\varphi_{\varepsilon}, x\right), \psi(x)\right\rangle=0 .
$$

By Definition 2, this proves (3) for $x \in \mathbb{R}$ and the result in the case of several variables follows directly from Theorem 1 .

On replacing $x$ by $-x$, interchanging $a$ and $b$, and taking into account that $\delta^{(p)}(-x)=(-1)^{|p|} \partial^{p} \delta(x)$ for $x \in \mathbb{R}^{m}$, we obtain the second equation in (4). This completes the proof.

Finally, these consequences follow from Theorem 2 when restricted to the cases: $b=0$ and $a=0$, respectively.

Corollary 1. . For each $a, b \in \Omega^{m}, b>-2$, and each $p \in \mathbb{N}_{0}^{m}$ these balanced products hold in the algebra $\mathcal{G}\left(\mathbb{R}^{m}\right)$ :

$$
\widetilde{\chi_{+}^{a}} \cdot \widetilde{\chi_{-}^{-a}}-\widetilde{\chi_{-}^{p+1}} \cdot \widetilde{\delta^{(p)}}(x) \approx 0, \widetilde{\chi_{+}}{ }^{b+p+1} \cdot \widetilde{\delta^{(p)}}(x)-\widetilde{\chi_{-}^{b}} \cdot \widetilde{\theta}(x) \approx 0 .
$$




\section{References}

[1] J.-F. Colombeau, New Generalized Functions and Multiplication of Distributions, North Holland Math. Studies 84, Amsterdam (1984).

[2] B. Damyanov, Mikusiński type products of distributions in Colombeau algebra, Indian J. Pure Appl. Math., 32, No 3 (2001), 361-375.

[3] B. Damyanov, Balanced Colombeau products of the distribution $x_{ \pm}^{-p}$ and $x^{-p}$, Czechoslovak Math. J., 55 (2005), 198-201.

[4] B. Damyanov, Results on generalized models and singular products of distributions in the Colombeau algebra $\mathcal{G}(\mathrm{R})$, Comment. Math. Univ. Carolinae, 56 (2015), 145-157.

[5] J. Mikusiński, On the square of the Dirac delta-distribution, Bull. Acad. Pol. Ser. Math. Astron. Phys., 43 (1966), 511-513.

[6] M. Nedeljkov, M. Oberguggenberger, Ordinary differential equations with delta function terms, Publ. Inst. Math. Beograd, 91 (2012), 125-135. 